nephron

Practice
Nephron

DOI: $10.1159 / 000490118$
Received: May 15, 2018

Accepted after revision: May 16, 2018

Published online: June 15, 2018

\title{
What is the Role of Soluble Urokinase-Type Plasminogen Activator in Renal Disease?
}

\author{
Moin A. Saleem \\ Bristol Royal Hospital for Children, University of Bristol, Bristol, UK
}

A comment on Hayek, et al: A tripartite complex of suPAR, APOL1 risk variants and av $\beta 3$ integrin on podocytes mediates chronic kidney disease. Nat Med 2017;23:945-953.

\section{Keywords}

Focal segmental glomerular sclerosis - Renal disease ·

Soluble urokinase-type plasminogen activator

\begin{abstract}
Context: Soluble urokinase-type plasminogen activator (suPAR) is an inflammatory signal with pleiotropic biological effects depending on context and post-translational modifications. Recently, [Hayek, et al: Nat Med 2017;23:945-953] it has been found that there is a link between suPAR and renal disease in several guises, and a key question is whether it is a driver or a marker of renal disease, and if so of which types of kidney damage. Subject of Review: Circulating suPAR has been postulated to cause acute proteinuric kidney disease, specifically focal segmental glomerulosclerosis (FSGS), though both the animal models and clinical data in the original reports have been challenged. More recently, suPAR levels are linked to the risk of progression of chronic kidney disease (CKD) by directly activating signaling pathways in the podocyte or as a sensitive early biomarker. Second Opinion: The evidence for suPAR as a driver of FSGS proteinuria is not yet established, and more needs to be done, particularly by measuring different circulating suPAR fragments and identi-
\end{abstract}

\section{KARGER}

(c) 2018 S. Karger AG, Basel

E-Mail karger@karger.com

www.karger.com/nef fying "second hits," to clarify if any role exists. The evidence for suPAR as a sensitive biomarker for CKD progression is currently stronger, and needs further independent validation as well as prospective serial suPAR measurements in CKD cohorts. Whether it is a surrogate marker for ongoing inflammatory drivers of kidney disease, or has direct mechanistic effects in podocytes will depend on further studies, as well as trials of suPAR removal or direct blockade of downstream pathways.

(c) 2018 S. Karger AG, Basel

\section{Introduction}

Soluble urokinase-type plasminogen activator (suPAR) is a soluble receptor derived from the cell membrane tethered receptor UPAR. UPAR is enzymatically cleaved from cell surfaces to become suPAR and is known to be a circulating biomarker closely linked to inflammation, organ damage, and immune activation in a variety of different disease states.

More recently, suPAR has been linked to renal disease, first as a potential circulating bioactive factor causing focal segmental glomerulosclerosis (FSGS), and then as a 
predictor of the rate of progression in prevalent chronic kidney disease (CKD), possibly linked to its functional interaction with apolipoprotein 1 (APOL1).

In this review, I delineate the biology of suPAR and what is known currently about its role in kidney disease, discuss aspects we still need to elucidate, and explore how this intriguing molecule could take us forward in renal medicine.

\section{Biological Function of Urokinase and uPAR}

\section{UPA (Urokinase) and Its Receptor UPAR}

Urokinase, also known as the urokinase-type plasminogen activator (uPA), is a serine protease, synthesized in large amounts in the kidney [1] and present in blood and extracellular matrix (ECM). Its main substrate, plasminogen, is an inactive form of the serine protease plasmin. The activation of plasmin by the proteolytic activity of uPA on plasminogen leads to thrombolysis or matrix degradation (depending on the environment). Thus, uPA, apart from its thrombolytic properties, is also able to affect cell migration, adhesion, and mitosis. In the ECM, uPA binds to its cellular receptor, uPAR. uPAR is a membrane-bound $45-55 \mathrm{kDa}$ protein with 3 extracellular domains (D1, D2, and D3) linked to a glycosylphosphatidylinositol (GPI) anchor in the cell membrane [2].

At the cell surface, uPAR also interacts with several soluble and membrane proteins other than uPA, for example, vitronectin, integrins, and thrombospondin, indicating important downstream cellular functions of this system independent of plasminogen activation [2].

The different domains of uPAR are notable. The region linking $\mathrm{D} 1$ and $\mathrm{D} 2 \mathrm{D} 3$ in uPAR is prone to cleavage by several proteases including physiologically relevant enzymes such as neutrophil elastase, plasmin, and uPA itself [3]. The amino terminal domain of uPAR (D1) contains the main uPA-binding site [4], whereas the next 2 domains of UPAR (D2D3) bind the ECM protein vitronectin [5]. However, full-size uPAR is required to reach high affinity in both interactions [6].

\section{Generation of suPAR}

When uPAR is cleaved by proteases from the cell surface (at the GPI anchor), a soluble form of uPAR (suPAR) is released [7]. The majority of circulating suPAR is generated by GPI-anchor cleavage, thought to be catalyzed by GPI-specific phospholipase-D (GPI-PLD). More recently, the transmembrane phospholipase-C GDE3 was shown to be a specific enzyme for uPAR cleavage [8]. Other proteases, including cathepsin $\mathrm{G}$ and phosphatidylinositol-specific phospholipase-C, are known to also cleave the GPIanchor, and thus suPAR generated by GPI-anchor cleavage is not only regulated by one kind of protease but potentially by a mixture of various proteases. Full-length suPAR (suPAR D1-3) can be cleaved into another 2 soluble forms with different biologic properties, suPAR D2D3 and suPAR DI [7]. Therefore, 3 fragments are possible $\mathrm{D} 1$,which is rapidly cleared, and not detected in plasma [9]; D1-3 (full length); and D2D3.

\section{Systemic Functions of suPAR}

It is hypothesized that the production of this active (D2D3) form of suPAR by activated neutrophils in sites of acute inflammation contributes to the recruitment of monocytes to these sites during an inflammatory response [10]. The function of this cleaved receptor has been studied further and it has now been well documented that suPAR D2D3 is a chemotactic agent $[11,12]$. There seems to be very strong evidence that suPAR D2D3 is mediating a chemotactic effect on immunologically active cells; whether it is promoting the immune response or merely a result of an increased activation level of the immune system is still not clear.

In healthy individuals, suPAR levels are quite stable in both blood and urine and suPAR levels in urine, adjusted according to urine creatinine and correlate positively and strongly with plasma suPAR-levels. Also, circadian suPAR plasma-concentrations (measured $24 \mathrm{~h}, 20 \mathrm{~min}$ intervals) appear to be stable [13].

\section{Local (Cellular) Actions of uPAR/suPAR}

UPAR appears to be important in promoting cell migration. uPAR is able to move on the cell surface [14] and localize into aggregates on the leading edge of migrating cells $[15,16]$ and can regulate the activation state of integrins [17].

suPAR is believed to have inhibiting properties on uPAR-dependent adhesion, as it is soluble and therefore not able to direct integrin and vitronectin molecules to the focal contacts $[9,18]$.

UPAR has dramatic effects on the promotion of cell motility, independent of uPA binding, via an interaction with vitronectin, initiating a Rac-dependent actin reorganization [7].

Importantly, the different fragments of suPAR and the cell type affect the direction of signaling induced by suPAR. In cells where uPA-uPAR is active, full-length suPAR acts to downregulate promigratory signaling, 
probably by competitive displacement of the uPA-uPAR complex from signaling adaptor proteins [19]. Proteolytic cleavage of suPAR to the D2D3 form increases its signaling activity, likely because the cleaved form has a similar signaling ability like that of uPA. This could be important in interpreting the effects of suPAR fragments on podocyte migration (see below).

\section{A Role for suPAR in Renal Biology}

\section{Role in Primary Proteinuric Disease (Notably FSGS)}

There is concrete evidence from in vitro and animal models that UPAR binds to and activates b3 integrin on the podocyte cell surface, leading to changes in podocyte motility and proteinuria [20]. The first specific action of the UPAR system on renal glomerular cell signaling was the demonstration that uPAR expression on podocytes mediates cell migration, via avb3 integrin and Rac1, and mediates lipopolysaccharide (LPS)-induced proteinuria in mice [20]. Subsequently, the same group demonstrated that circulating suPAR activated avb3 integrin on podocytes, causing proteinuria in mouse models. Concomitantly they found higher levels of circulating suPAR in a high proportion of patients with FSGS compared to other glomerular diseases [21].

Following this landmark report, many groups have attempted to reproduce the clinical findings in other cohorts. The evidence has been reviewed elsewhere (e.g., $[22,23])$ and definitive proof of circulating suPAR as a predictive or ongoing clinical biomarker for FSGS remains elusive. The difficulty in assessing suPAR as a biomarker for FSGS has been compounded by the confounding effect of suPAR levels being negatively correlated with GFR, with FSGS often diagnosed in stages of CKD where GFR is already reduced.

There have also been studies to extend the experimental evidence that suPAR is a causative factor. These have not been able to replicate the finding that suPAR causes acute proteinuria (see below [24, 25]).

suPAR has also been studied in renal biopsy samples. $\mathrm{Wu}$ et al. [26] found higher suPAR levels in common biopsy-proven kidney diseases, especially in diabetic nephropathy $(\mathrm{DN})$ correlating with increasing proteinuria. Upregulated expression of suPAR in renal tissue was noted in most of the disease samples. Serum suPAR gradually increased with severity and staging of DN. In addition, levels of suPAR were linearly related to eGFR and to the amount of proteinuria in patients with various kidney diseases. In this study, after adjusting for age and multi- variate linear regression of eGFR and proteinuria, the suPAR levels are still significantly negatively related to eGFR $(r=-0.579, p<0.001)$ and positively related to proteinuria $(r=0.459, p<0.001)$.

There appears to be a multifactorial relationship between circulating suPAR and renal disease in general, with levels elevated by decreasing GFR, inflammation, and also some evidence of an independent relationship to proteinuria, albeit not consistently yet shown for any one type of glomerular disease.

\section{Does High Circulating suPAR Cause Proteinuria?}

\section{Mouse Models}

The original evidence was demonstrated in the mouse model of LPS proteinuria. The study by Wei et al. [21] used the uPAR knockout mouse (PLAUR ${ }^{--}$) to reintroduce recombinant full-length suPAR via tail vein injection, and reported the induction of proteinuria (in LPS mice). A suPAR variant D1D2 was also generated in that study (not a naturally occurring variant) and expressed in WT mice by electroporation, causing rapid proteinuria and histological features suggestive of glomerular scarring.

Similarly, in a follow-up paper, the authors demonstrated that bone marrow-derived cells from LPS-induced proteinuric mice produce excess suPAR, and when transferred to PLAUR ${ }^{-/-}$mice result in proteinuria [27].

While the original studies by Wei et al. [21] showed that full-length suPAR can cause rapid proteinuria in PLAUR null mice but not wild-type (WT) mice, attempts to replicate acute proteinuria in WT mice have not been successful so far. Cathelin et al. [24] confirmed the absence of proteinuria following full-length suPAR exposure in WT mice over a few weeks. This was despite the demonstration of suPAR deposition in the kidney. Spinale et al. [25] used constitutive overexpression of suPAR (full length) in WT mice, using a transgenic model, wherein suPAR is inducibly overexpressed by the liver. Again, this did not result in proteinuria.

Full-length recombinant suPAR did result in some increase in proteinuria in WT mice induced by anti-CD40 autoantibodies + recurrent FSGS plasma [28]. Furthermore, Hahm et al. [27] showed that full length suPAR expression from transgenic adipocytes causes very mild but significantly increased proteinuria (around $150 \mathrm{mg} / \mathrm{g}$ ) in mice exposed to high levels of suPAR for several months. 
This implies that the context of suPAR overexpression is important, and the presence of high suPAR levels alone is not enough to generate podocyte damage.

\section{Human Studies}

It has not yet been established whether suPAR in humans directly causes proteinuria. The natural human experiment of paroxysmal nocturnal hematuria, $\mathrm{PNH}$, supports the concept discussed above that high suPAR levels alone are not enough to initiate proteinuria. Here the defect is in the GPI anchoring region of uPAR, resulting in very high ongoing levels of circulating suPAR (up to $8,000 \mathrm{pg} / \mathrm{mL}$ ), but not primarily proteinuria [29].

The renal damage in this disease is caused primarily by unregulated microvascular thrombotic events, with eventual cortical infarcts [30].

Another related question in human disease is as follows: what is the source of increased circulating suPAR? The mouse studies mentioned above suggest that a bone marrow subpopulation could be responsible, though the equivalent bone marrow markers are not present in humans; so the findings are difficult to confirm in human disease. There are also interesting data showing that UPAR is significantly upregulated in a variety of different kidney diseases, including minimal change disease, DN and immunoglobulin A nephropathy, in glomeruli and tubules, alongside increased suPAR in most of these diseases [26], raising a possibility that some of the increased suPAR production could arise from the kidney itself.

\section{Clinical Correlation of suPAR Levels in Proteinuric Kidney Disease}

The original study by Wei et al. [21] demonstrated plasma levels of suPAR significantly higher in patients with FSGS (compared to other renal conditions) both pre- and post-transplantation, with higher levels in recurrence post-transplant, compared to those who did not recur. The authors defined a level of $3,000 \mathrm{pg} / \mathrm{mL}$ as a cutoff value that implied a high probability of diagnosing FSGS. The problem has been that many subsequent studies, amounting to a large cohort of patients, have not replicated this association. Some are supportive, while others show no correlation at all, testing a variety of renal conditions including genetic podocyte disease and normal controls. The conclusion of several of these studies is that elevated suPAR correlates closely with eGFR, though other studies have shown this is not the only factor contrib- uting to elevated suPAR in kidney disease. For example, one study demonstrates suPAR levels correlate with the degree of proteinuria independent of eGFR, though not just in FSGS patients [26], and another showed high suPAR correlates with increased cardiovascular mortality in CKD patients, over and above the effects of eGFR loss [31].

Levels of suPAR in FSGS that are deemed to correlate with proteinuria are similar to levels in the normal population that do not have (nephrotic range) proteinuria. The average level in a population is quoted at $3,400 \mathrm{pg} / \mathrm{mL}$ (higher than the cut-off level in the original Wei et al. [21] paper), and in an intensive care unit averages $10,000 \mathrm{pg} /$ $\mathrm{mL}$ (www.suparnostic.com/index.php/products/resinterpretation). Therefore, at the levels quoted, there should be at the least a second hit in FSGS that results in filtration barrier breakdown. As on date, this has not been investigated in the suPAR literature. Moreover, that "hit" needs to be present in the transplant recipient, considering that recurrence is not specific to features of the donor kidney, therefore making it difficult to implicate podocyte susceptibility.

\section{Effect of Distinct suPAR Fragments}

A significant limitation of the human studies to measure suPAR levels is that the available ELISA kits do not differentiate different fragments. Additionally, there are glycosylation variants of suPAR that have not been independently studied in renal disease. Thus, if there are different biological functions of distinct fragments to drive specific renal pathology, this has not yet been sufficiently investigated.

Different ratios of suPAR fragments may relate to different levels of protease activity, some proteases cleaving the linker region (resulting in D1 and D2-3) and some cleaving the GPI anchor (resulting in D1-3).

All of the studies, including the original by Wei et al. [21], measure suPAR by commercial ELISA, which detects all fragments without being able to distinguish if any fragment predominates. This leaves an open question about whether FSGS, or other proteinuric conditions, might display distinct patterns of circulating suPAR fragments, which could have more specific biological effects. As mentioned above, it has been shown that the D2D3 fragment has opposing effects on cell migration to the full length D1-3 fragment in cells with uPA/uPAR activity [19]. To date, there is no clinical evidence of different fragments predominating in FSGS. In theory, it would be very plau- 
sible to test for suPAR fragments from recurrent FSGS patients on a Western blot, using plasma samples immunoprecipitated with antibodies to the different fragments [4].

\section{Role of suPAR in Progression of Chronic Disease}

\section{Could suPAR Be Worsening Fibrosis by Decreased}

Fibrin Removal?

The fibrinolytic pathway has well-recognized anti-fibrotic effects. Fibrin forms within the organ due to a leakage of plasma from damaged vasculature and activation of the coagulation cascade. The extravascular fibrin is not cleared in a timely fashion because of a marked increase in the expression of plasminogen activation inhibitor-1 relative to urokinase-type plasminogen activator $[32,33]$.

The uPA/uPAR system is therefore involved in a number of processes that are associated with turnover of the ECM. Plasmin generates matrix metalloproteases, initiating chemotaxis and extravasation of inflammatory cells, and also permitting penetration of those cells into the tissue. Conversely, the activated fibrinolytic system could play a role in preventing fibrosis by diminishing the accumulation of matrix via this induction of plasmin and matrix metalloproteases.

It is also suggested that circulating suPAR can influence the activity of uPA by acting as a scavenger for uPA in the circulation, acting as a competitive inhibitor of uPAR, thereby limiting uPA effects on attenuating tissue fibrin deposition. This could only occur for full length (D1-D3) suPAR, as the D1 region is needed for uPA binding [34]. Thus, the question of suPAR potentially modulating renal fibrosis is a very pertinent one.

This can be seen in animal models and human disease, for example, overexpression of uPA in lungs of mice protects against pro-fibrotic damage [35], and high suPAR levels are associated with slow coronary flow, where epicardial coronary flow is slow (without obstruction) and may be a sign of microcirculatory or endothelial damage [36].

\section{suPAR is Proposed as a Risk Predictor for Progression} of $C K D$

Following the description of suPAR as a potential FSGS "circulating factor" came the publication of a major paper delineating a relationship between suPAR levels at baseline assessment and progression of CKD [37].

The patients tested were recruited at the time of cardiac catheterization, with approximately a third having normal eGFR at baseline (enrolment). The higher the lev- el of suPAR at baseline, the greater the subsequent decline in eGFR. Those with normal eGFR at baseline had the greatest suPAR-related decline in eGFR. Thus, initial suPAR level appears to be a sensitive predictor of rate of decline of renal function in this cardiac disease population.

In order to test whether this correlated with inflammatory markers in the same population, the authors went on to examine the relationship between a range of inflammatory markers and risk of CKD progression [38], and found only a weak correlation, with suPAR remaining independently predictive.

The authors subsequently examined 2 European pediatric cohorts with established CKD of varying etiologies and reported a similar pattern of more rapid decline associated with higher baseline suPAR levels [39]. Importantly, a later Swedish study reported a significant correlation between baseline suPAR levels and longitudinal decline in eGFR, higher incidence of CKD and hospitalization due to impaired kidney function in a cohort of healthy middle-aged participants [40]. None of these studies to date have reported whether suPAR levels remain elevated throughout the course of disease progression, which would need to be measured longitudinally.

There is also some data on suPAR as a potential early biomarker for acute kidney injury (AKI), suggesting patients who develop AKI post cardiac surgery have a higher level of suPAR preoperatively [41].

\section{sUPAR is Linked to APOL1 Biology}

Following on from this, the same group that first described the association between CKD progression and suPAR sought to identify a synergy between suPAR and the established APOL1 risk variants for CKD [42]. They looked at the Emory cohort initially described, and a second cohort, the African American Study of Kidney Disease and Hypertension. In the first, but not the second cohort, high suPAR levels correlated with the high-risk APOL1 genotype [43].

SuPAR levels were a substantial feature of the association between APOL1 genotype and subsequent eGFR decline. Higher baseline suPAR levels were associated with a significantly greater yearly decline in kidney function in patients with 2 APOL1 risk alleles than those in patients with one or zero alleles.

A biological basis for this observation was presented, where it was elegantly shown that suPAR, APOL1, and avb3 integrin interact, but the higher risk G1 and G2 allelelic variants of $A P O L 1$ interact more strongly with activated avb3 integrin on the cell surface. Thus, a mecha- 
nism of $A P O L 1$ risk variant association with more rapid progression of CKD could be via its interaction with suPAR and avb3 integrin on the podocyte cell surface.

\section{Is There a Unifying Role of suPAR in Kidney Disease?}

The overall question remains as to whether suPAR is (1) a specific driver of kidney (podocyte) damage and causes/ exacerbates a variety of pathologies from FSGS to CKD to AKI, or (2) whether it is a surrogate biomarker of background systemic pathology, particularly inflammation, that is driving renal damage in certain clinical situations.

From the perspective of point number (1) made above, there is compelling experimental evidence of a biological effect of suPAR on podocyte dynamics, particularly via the activation of the $\mathrm{b} 3$ integrin signaling pathway, and also modulated by APOL1. Given the clear link between APOL1 genotype and CKD progression (both in FSGS and other forms of kidney disease), a theoretical basis for increased circulating suPAR exacerbating CKD progression has been elucidated. The next step is to take the initial link between baseline suPAR levels and progression and establish clinical correlative links between sustained suPAR levels and CKD progression. This would be required to support a functional role for suPAR in driving progressive damage.

The field has been confused by conflicting studies on suPAR levels in FSGS and needs clarity. The best way to approach this is by optimal stratification of patients according to current knowledge, combined with greater clarification of suPAR fragments being measured. Stratification should aim to define those patients with known "circulating factor disease" according to clinical criteria. This involves the identification of patients who definitely do or do not have circulating factor disease (CFD) by: (i) diagnosis of monogenic disease by gene panel analysis for all known SRNS genes, and a detailed family history (in cases of as yet undiscovered genes), and (ii) identification of patients with a definite/very highly likely CFD, that is, initial steroid sensitivity, and development of post-transplant recurrence [44]. With those 2 categories as the negative and positive standards, other forms of FSGS/SRNS can be assessed according to suPAR levels found. For example, in the subset of children with primary steroid resistance and negative gene panel/exome testing, we have demonstrated that the rate of post-transplant recurrence is $50 \%$ [ 45 ]. Therefore, at least $50 \%$ of children in this category would be predicted to have high suPAR levels pretransplant, and have levels comparable to the positive control group. Interestingly at least one previous study has reported high suPAR levels in children with monogenic (non-circulating factor) SRNS, thereby further complicating interpretation [46]. Ideally all of these studies should also include measurement of suPAR fragments (D1-3 and D2-3) to determine if there is a differential effect/pattern of either fragment.

From the perspective of point number (2) made above, that suPAR is a biomarker or surrogate marker for a systemic response, one likely biological function is as an inflammatory modulator. There exists a wealth of experimental and observational data demonstrating that suPAR is elevated in numerous conditions of systemic inflammation or infection and associated with prognosis. This should therefore not be discounted, even if simply as a potential confounder in situations where suPAR could otherwise directly be driving kidney damage.

SuPAR is a prognostic marker in various non-CKD diseases, including cardiovascular disease, where it is strongly linked to inflammation. Therefore, the most important alternative mechanism to be excluded for suPAR in CKD is that it is a marker of presence or severity of inflammatory disease, which is independently driving the severity of the kidney disease (and hence progression). This is supported by a report showing that patients with stages I and II CKD secondary to glomerulonephritis had higher suPAR levels according to the severity (on biopsy) of their glomerulonephritis [47].

Interestingly, a recent study in the US million veterans' cohort associates increased baseline monocyte count with risk of progression of CKD [48]. This was a graded correlation, with higher levels of monocytes associated with a greater rate of decline in renal function, very similar to the suPAR correlation. Clearly monocyte count can be a marker of chronicinflammation, and therefore it strengthens the association between the degree of inflammation and risk of renal function decline [49].

There are at least 2 potential scenarios for suPAR in inflammatory renal disease. Could suPAR in the kidney be promoting inflammation, chemotaxis in particular, and directly amplifying the underlying inflammatory state? Or is it merely marking the systemic (and local) inflammatory response that is a primary driver of certain chronic renal diseases (e.g., DN, lupus nephritis, IgAN)? In either scenario, it could be part of an inflammatory milieu that links cardiovascular disease to proteinuric renal disease.

Nevertheless, as long as the data are consistent in other cohorts, this still renders suPAR a valuable prognostic marker of progression, a critically required biomarker for reliably testing the effect of interventional new compounds (e.g., antifibrotic agents).

If suPAR is a mechanistic driver of renal damage, causing proteinuria either acutely or chronically perhaps via an 
APOL1 mediated pathway, the clinical confirmation could come from the removal of suPAR, once this technology is available. The most acute and rapid way of testing this hypothesis would be in patients with post-transplant recurrence of FSGS, though this does not specifically address the link to CKD. In CKD, the reduction of suPAR over a long period of time would be far more challenging. Alternative strategies would be to inhibit the downstream pathways in podocytes, for example, inhibition of a5b3 integrin signaling with novel compounds such as Cilengitide (a peptide molecule inhibitor of av integrin).

If it is to be postulated that the mechanism of suPAR causing proteinuria in FSGS is common with the mechanism linking suPAR to CKD progression, then the common pathway (given current knowledge) could be via b3 integrin activation. The anomalies lie in the differences in clinical presentation, one being of acute and massive proteinuria, the other being progressive fibrotic damage with or without proteinuria. Both have similar levels of suPAR. Clearly other modifying pathways would need to be discovered to explain these differences.

\section{Conclusion and Author's Opinion}

It is clear that suPAR is not the (sole) circulating factor for FSGS, but potentially a fragment is having an effect in a subset of patients, with at least one "second hit" necessary, which has not yet been found. This could potentially be a cytokine, as part of an inflammatory response [50], but alternatively suPAR could simply be a marker of an underlying inflammatory state that is driving subsets of disease by another mechanism.

Overall therefore, the view of suPAR in renal disease can be viewed from the perspective of (1) a potential cir- culating factor in SRNS/FSGS and (2) a biomarker and/ or driver of accelerated renal damage in CKD.

For (1), the majority view is that a role for suPAR as an acute proteinuric factor should be set aside, until and unless further clinical evidence is gathered. In many cohorts tested to date, most are negative or equivocal at best in supporting a distinct association between suPAR levels (as currently measured) and active disease. More evidence is also needed to ascertain if distinct suPAR fragments are associated with disease activity, in a more specific and reproducible manner.

For (2), the evidence is building that there is a correlation between suPAR levels and risk of progression of $\mathrm{CKD}$. One key question to now ascertain is whether this is a correlative biomarker, measuring coincident inflammatory activity in particular, or whether suPAR is a mechanistic driver of renal damage. This could be by biological effects on podocyte migratory pathways, with key evidence demonstrating an effect on the APOL1/b3 integrin signaling axis, or by more general effects as a scavenger for uPA, thereby limiting aspects of chronic fibrosis. It may be that a combination of the above factors explains the link seen between suPAR and CKD progression.

SuPAR could differentiate between inflammatory and non-inflammatory causes of $\mathrm{CKD}$, and this needs more nuanced investigation. Overall, this yields suPAR to be a promising biomarker for CKD progression, which needs further validation, particularly by longitudinal measurements and correlation, and potentially also as a target for therapy.

\section{Disclosure Statement}

The author has no conflicts of interest to declare.

\section{References}

1 Sappino AP, et al: Sites of synthesis of urokinase and tissue-type plasminogen activators in the murine kidney. J Clin Invest 1991;87:962-970.

$\checkmark 2$ Thuno M, Macho B, Eugen-Olsen J: suPAR: the molecular crystal ball. Dis Markers 2009; 27:157-172.

3 Behrendt $\mathrm{N}$, et al: The ligand-binding domain of the cell surface receptor for urokinase-type plasminogen activator. J Biol Chem 1991;266: 7842-7847.

4 Sidenius N, et al: Shedding and cleavage of the urokinase receptor (UPAR): identification and characterisation of uPAR fragments in vitro and in vivo. FEBS Lett 2000;475:52-56.
5 Wei Y, et al: Identification of the urokinase receptor as an adhesion receptor for vitronectin. J Biol Chem 1994;269:3238032388.

6 Ploug M, et al: Photoaffinity labeling of the human receptor for urokinase-type plasminogen activator using a decapeptide antagonist. Evidence for a composite ligand-binding site and a short interdomain separation. Biochemistry 1998;37:3612-3622.

7 Hillig T et al: A composite role of vitronectin and urokinase in the modulation of cell morphology upon expression of the urokinase receptor. J Biol Chem 2008;283:15217-15223.
8 van Veen M, et al: Negative regulation of urokinase receptor activity by a GPI-specific phospholipase $\mathrm{C}$ in breast cancer cells. Elife 2017;6:e23649.

9 Mustjoki S, et al: Soluble urokinase receptor levels correlate with number of circulating tumor cells in acute myeloid leukemia and decrease rapidly during chemotherapy. Cancer Res 2000;60:7126-7132.

10 Pliyev BK: Activated human neutrophils rapidly release the chemotactically active D2D3 form of the urokinase-type plasminogen activator receptor (uPAR/CD87). Mol Cell Biochem 2009;321:111-122. 
11 Alfano M, et al: Urokinase-urokinase receptor interaction mediates an inhibitory signal for HIV-1 replication. Proc Natl Acad Sci U S A 2002;99:8862-8867.

12 Fazioli F, et al: A urokinase-sensitive region of the human urokinase receptor is responsible for its chemotactic activity. EMBO J 1997;16: 7279-7286.

13 Gozdzik W, et al: Unchanged plasma levels of the soluble urokinase plasminogen activator receptor in elective coronary artery bypass graft surgery patients and cardiopulmonary bypass use. PLoS One 2014;9:e98923.

14 Myohanen HT, et al: Distribution and lateral mobility of the urokinase-receptor complex at the cell surface. J Histochem Cytochem 1993; 41:1291-1301.

15 Estreicher A, et al: The receptor for urokinase type plasminogen activator polarizes expression of the protease to the leading edge of migrating monocytes and promotes degradation of enzyme inhibitor complexes. J Cell Biol 1990;111:783-792.

16 Duriseti S, et al: Antagonistic anti-urokinase plasminogen activator receptor (uPAR) antibodies significantly inhibit uPAR-mediated cellular signaling and migration. J Biol Chem 2010;285:26878-26888.

$\checkmark 17$ Kugler MC, Wei Y, Chapman HA: Urokinase receptor and integrin interactions. Curr Pharm Des 2003;9:1565-1574.

18 Furlan F, et al: The soluble D2D3(88-274) fragment of the urokinase receptor inhibits monocyte chemotaxis and integrin-dependent cell adhesion. J Cell Sci 2004;117:29092916.

19 Jo M, et al: Soluble urokinase-type plasminogen activator receptor inhibits cancer cell growth and invasion by direct urokinase-independent effects on cell signaling. J Bio Chem 2003;278:46692-46698.

20 Wei C, et al: Modification of kidney barrier function by the urokinase receptor. Nat Med 2008;14:55-63.

21 Wei C, et al: Circulating urokinase receptor as a cause of focal segmental glomerulosclerosis. Nat Med 2011;17:952-960.

22 Maas RJ, Deegens JK, Wetzels JF: Serum suPAR in patients with FSGS: trash or treasure? Pediatr Nephrol 2013;28:1041-1048.

23 Wada T, Nangaku M: A circulating permeability factor in focal segmental glomerulosclerosis: the hunt continues. Clin Kidney J 2015; 8:708-715.

24 Cathelin D, et al: Administration of recombinant soluble urokinase receptor per se is not sufficient to induce podocyte alterations and proteinuria in mice. J Am Soc Nephrol 2014; 25:1662-1668.

25 Spinale JM, et al: A reassessment of soluble urokinase-type plasminogen activator receptor in glomerular disease. Kidney Int 2015;87: 564-574.

$26 \mathrm{Wu}$ CZ, et al: Urokinase plasminogen activator receptor and its soluble form in common biopsy-proven kidney diseases and in staging of diabetic nephropathy. Clin Biochem 2015; 48:1324-1329.

27 Hahm E, et al: Bone marrow-derived immature myeloid cells are a main source of circulating suPAR contributing to proteinuric kidney disease. Nat Med 2017;23:100-106.

28 Delville M, et al: A circulating antibody panel for pretransplant prediction of FSGS recurrence after kidney transplantation. Sci Transl Med 2014;6:256ra136.

29 Ploug M, et al: A soluble form of the glycolipid-anchored receptor for urokinase-type plasminogen activator is secreted from peripheral blood leukocytes from patients with paroxysmal nocturnal hemoglobinuria. Eur J Biochem 1992;208:397-404.

30 Clark DA, et al: The kidneys in paroxysmal nocturnal hemoglobinuria. Blood 1981;57: 83-89.

31 Meijers B, et al: Soluble urokinase receptor is a biomarker of cardiovascular disease in chronic kidney disease. Kidney Int 2015;87: 210-216.

32 Bertozzi P, et al: Depressed bronchoalveolar urokinase activity in patients with adult respiratory distress syndrome. N Engl J Med 1990; 322:890-897.

- 33 Idell S et al: Local abnormalities in coagulation and fibrinolytic pathways predispose to alveolar fibrin deposition in the adult respiratory distress syndrome. J Clin Invest 1989;84: 695-705.

34 Masucci MT, Pedersen N, Blasi F: A soluble, ligand binding mutant of the human urokinase plasminogen activator receptor. J Biol Chem 1991;266:8655-8658.

35 Sisson TH, et al: Inducible lung-specific urokinase expression reduces fibrosis and mortality after lung injury in mice. Am J Physiol Lung Cell Mol Physiol 2002;283:L1023L1032.

36 Emrah A, et al: Increased circulating soluble urokinase-type plasminogen activator receptor (suPAR) levels in patients with slow coronary flow. Arch Med Sci Atheroscler Dis 2016;1:e53-e59.
37 Hayek SS, et al: Soluble urokinase receptor and chronic kidney disease. N Engl J Med 2015;373:1916-1925.

38 Hayek SS, et al: Cardiovascular disease biomarkers and suPAR in predicting decline in renal function: a prospective cohort study. Kidney Int Rep 2017;2:425-432.

39 Schaefer F, et al: Association of serum soluble urokinase receptor levels with progression of kidney disease in children. JAMA Pediatr 2017; 171:e172914

-40 Schulz CA, et al; Soluble irokinase-type plasminogen activator receptor (suPAR) and impaired kidney function in the populationbased Malmo Diet and Cancer Study. Kidney Int Rep 2017;2:239-247.

41 Mossanen JC, et al: Elevated soluble urokinase plasminogen activator receptor and proenkephalin serum levels predict the development of acute kidney injury after cardiac surgery. Int J Mol Sci 2017; 18:pii:E1662 .

42 Parsa A, et al: APOL1 risk variants, race, and progression of chronic kidney disease. $\mathrm{N}$ Engl J Med 2013;369:2183-2196.

43 Hayek SS, et al: A tripartite complex of suPAR, APOL1 risk variants and alphavbeta3 integrin on podocytes mediates chronic kidney disease. Nat Med 2017;23:945-953.

44 Ding WY, et al: Initial steroid sensitivity in children with steroid-resistant nephrotic syndrome predicts post-transplant recurrence. J Am Soc Nephrol 2014;25:1342-1348.

45 Bierzynska A et al: Genomic and clinical profiling of a national nephrotic syndrome cohort advocates a precision medicine approach to disease management. Kidney Int 2017;91: 937-947.

46 Wei C et al: Circulating suPAR in two cohorts of primary FSGS. J Am Soc Nephrol 2012;23: 2051-2059.

47 Quaglia M, Musetti C, Cantaluppi V: Soluble urokinase receptor and chronic kidney disease. N Engl J Med 2016;374:890.

48 Bowe B, et al: Association between monocyte count and risk of incident CKD and progression to ESRD. Clin J Am Soc Nephrol 2017; 12:603-613.

49 Heine GH, et al: Monocyte subpopulations and cardiovascular risk in chronic kidney disease. Nat Rev Nephrol 2012;8:362-369.

50 Kim EY, Roshanravan H, Dryer SE: Changes in podocyte TRPC channels evoked by plasma and sera from patients with recurrent FSGS and by putative glomerular permeability factors. Biochim Biophys Acta 2017;1863: 2342-2354 